\title{
Outcome of Nicotine Replacement Therapy in Patients Admitted to ICU: A Randomized Controlled Double-Blind Prospective Pilot Study
}

\author{
Vikas Pathak MD, Iliana Samara Hurtado Rendon MD, Roxana Lupu MD, Nancy Tactuk MD, \\ Toyosi Olutade MD, Carolyn Durham PhD, and Richard Stumacher MD
}

\begin{abstract}
BACKGROUND: The effect of nicotine withdrawal in smokers admitted to the ICU is not well understood, so the role of nicotine replacement therapy (NRT) in those patients is controversial. OBJECTIVE: To determine whether NRT in ICU patients affects the need for sedatives/analgesics, ventilator days, and ICU stay. METHODS: In a 20-bed ICU, 40 subjects were randomized to either a $21 \mathrm{mg}$ nicotine patch or a placebo nicotine patch daily until either ICU discharge, transfer to a medical floor, or 10 weeks in the ICU. We collected data on sedatives/analgesics use during ICU stay and use and duration of mechanical ventilation . RESULTS: There were 27 male and 13 female subjects. The mean age was $57.4 \mathrm{y}$ in the intervention group and $52.5 \mathrm{y}$ in the control group. The mean Acute Physiology and Chronic Health Evaluation II score was 14.3 in the intervention group and 13.8 in the control group. The mean ICU stay was $4.5 \mathrm{~d}$ in the intervention group and $7 \mathrm{~d}$ in the control group. The mean number of days on ventilator was 1.9 in the intervention group and 3.5 in the control group. The number of days on sedation/analgesia was less in the intervention group than in the control group. CONCLUSIONS: Although ICU stay and ventilator days decreased numerically in this pilot study, statistically there was no beneficial effect from NRT. (International Standard Randomised Controlled Trial Register ISRCTN66928309) Key words: ICU; nicotine addiction; nicotine replacement therapy; smoking status. [Respir Care 2013;58(10):1625-1629. (C) 2013 Daedalus Enterprises]
\end{abstract}

\section{Introduction}

There is vast literature establishing nicotine addiction in people who smoke, and when smokers are admitted to ICUs they must cope with the effects of enforced nicotine

At the time of this research Dr Pathak was affiliated with the Department of Internal Medicine, St Barnabas Hospital, Bronx, New York. He is now affiliated with the Department of Pulmonary Disease and Critical Care Medicine, University of North Carolina School of Medicine, Chapel Hill, North Carolina. Drs Rendon, Lupu, Tactuk, and Olutade are affiliated with the Department of Internal Medicine; and Dr Stumacher is affiliated with the Intensive Care Unit/Pulmonary Medicine, St Barnabas Hospital, Bronx, New York. Dr Durham is affiliated with the University of North Carolina, Chapel Hill, North Carolina.

The authors have disclosed no conflicts of interest.

Correspondence: Vikas Pathak MD, School of Medicine, University of North Carolina, 130 Mason Farm Road, Chapel Hill NC 27599. E-mail: drvikaspathak@gmail.com.

DOI: $10.4187 /$ respcare.01791 withdrawal. ${ }^{1,2}$ The effects of nicotine withdrawal in smokers admitted to the ICU are not well understood. ${ }^{2}$ There are limited available data on nicotine replacement therapy (NRT) in hospitalized smokers, ${ }^{3}$ so the role of NRT in smokers admitted to ICUs remains controversial. ${ }^{4} \mathrm{We}$ conducted a placebo-controlled, double-blind, randomized, prospective pilot study to determine whether NRT in cigarette smokers in the ICU decreases the effects of nicotine withdrawal, the use of sedatives and analgesics, the number of ventilator days, or ICU stay. We report the preliminary results of our pilot study. We expect to complete the study over the course of the next few years, and will then report the complete study results.

\section{Methods}

Study Design and Subjects

This was a placebo-controlled, double-blind, randomized, pilot study done at St Barnabas Hospital, Bronx, New York, in a 20-bed mixed medical/surgical ICU. Following 
Table 1. Inclusion and Exclusion Criteria

\begin{tabular}{l}
\hline \hline Inclusion criteria \\
Subject admitted to intensive care unit \\
With or without ventilator \\
Informed consent from subject or delegate \\
Smoked $\geq 1$ pack of cigarettes per day \\
No contradiction to nicotine use \\
Exclusion criteria \\
Myocardial infarction in last 2 weeks \\
Unstable angina \\
Uncontrolled or serious arrhythmia \\
Severe allergic reaction
\end{tabular}

approval by our institutional review board, 40 subjects were enrolled: 20 in the intervention group, and 20 in the control group. Table 1 shows the inclusion and exclusion criteria. After providing informed consent, subjects were randomized to either the intervention (NRT) or control (placebo) group. Subjects in the intervention group had a $21 \mathrm{mg}$ nicotine patch applied to the skin within 48 hours of ICU admission. A new patch was applied daily until either discharge from the ICU, transfer to a general medical floor, or until 10 weeks had passed. If the subject was in the ICU for 10 weeks or longer, the weaning of nicotine was the same as in out-patient settings over 10 weeks. Subjects in the control arm had a sham (no nicotine) patch applied with the same frequency and duration. Subjects and their care providers were unaware of which type of patch they received.

\section{Data Collection}

Intake data regarding cigarette smoking, comorbidities, and reason for ICU admission were collected upon enrollment. We collected data on worst daily mean arterial pressure, heart rate, and use of vasopressors, sedatives, and analgesics during ICU stay, after the application of each patch. Use and duration of mechanical ventilation and ICU stay were also recorded. The investigators collecting the data were blinded as to whether a subject had a nicotine or placebo patch. The subjects were followed only through their ICU stay.

\section{Statistical Analysis}

Each subject's Acute Physiology and Chronic Health Evaluation (APACHE II) score was used as a matching variable between the treatment group and the control group. Discrete clinical characteristics (eg, history of acquired immunodeficiency syndrome, coronary artery disease, and cancers) were compared between the treatment and control groups with the McNemar test for paired data. Continuous clinical characteristics (eg, ICU stay, packs of

\section{QUICK LOOK}

\section{Current knowledge}

The effects of nicotine withdrawal in smokers admitted to intensive care are not well described, and the use of nicotine replacement therapy in those patients is controversial.

\section{What this paper contributes to our knowledge}

In this small pilot study, nicotine replacement therapy was associated with shorter duration of mechanical ventilation, shorter ICU stay, and reduced use of analgesics, but the differences were not statistically significant, and larger studies are required.

cigarettes smoked per day, and number of days on sedation) were compared with the Wilcoxon signed-rank test for paired data. Discrete variables are presented as number and percent. Continuous variables are presented as mean $\pm \mathrm{SD}$ or median. A $P$ value of $<.05$ was taken as a statistically significant difference. The analyses were carried out with statistics software (SAS, SAS Institute, Cary, North Carolina).

\section{Results}

We followed 40 subjects (20 intervention, 20 control) over a 6-month period (Table 2). Twenty subjects received mechanical ventilation (10 intervention, 10 control), and 20 did not (10 intervention, 10 control). There were 27 male and 13 female subjects; 20 subjects were Hispanic, 10 were African-American, and 10 were white. The mean age was 57.4 years in the intervention group and 52.5 years in the control group. The severity of illness was equivalent in the groups: the mean APACHE II score was 14.3 in the intervention group and 13.8 in the control group. The mean number of cigarette packs smoked per day was 1.2 in the intervention group and 1.0 in the control group. The duration of smoking was equivalent in the groups: $24.4 \mathrm{y}$ in the intervention group and $23.3 \mathrm{y}$ in the control group. All the subjects were admitted to the ICU from either a general medical floor or the emergency room. There was no cutoff as to when a subject from a general medical floor could join the study. Interestingly, all subjects but one were admitted from the emergency room. This subject (from the general medical floor), however, was admitted to the ICU within an hour of being on the general medical floor.

We verified that the subject transferred to the ICU from the general medical floor was not already using NRT. Subjects were admitted with various diagnoses in both 
Table 2. Characteristics of Subjects by Nicotine Replacement Therapy Status

\begin{tabular}{lrr}
\hline \hline & \multicolumn{2}{c}{$\begin{array}{c}\text { Nicotine Replacement Therapy, } \\
\text { no. (\%) }\end{array}$} \\
\cline { 2 - 3 } & Yes & No \\
\hline Ethnicity & & \\
$\quad$ Hispanic & $10(50)$ & $10(50)$ \\
African American & $5(25)$ & $5(25)$ \\
White & $5(25)$ & $5(25)$ \\
Ventilator & & $10(50)$ \\
Yes & $10(50)$ & $10(50)$ \\
No & $10(50)$ & \\
\hline
\end{tabular}

groups, and there was no significant difference in the admission diagnoses between the 2 groups. There were no differences in the reasons for intubation between the 2 groups; the reasons ranged from respiratory failure to airway protection to sepsis. The mean number of days on analgesics was $1.1 \mathrm{~d}$ in the intervention group and $2.1 \mathrm{~d}$ in the control group. The mean number of days on vasopressors was almost equal: $0.7 \mathrm{~d}$ in the intervention group and $0.5 \mathrm{~d}$ in the control group. The mean number of days on sedation was nearly double in the control group (2.7 d), compared to the intervention group (1.4 d). The mean ICU stay decreased by 2.5 days with NRT. In the intervention group the mean ICU stay was $4.5 \mathrm{~d}$, versus $7 \mathrm{~d}$ in the control group (Table 3). The mean ventilator days also decreased by 1.6 days with NRT use ( $1.9 \mathrm{~d}$ vs $3.5 \mathrm{~d})$. None of the subjects died while in the study.

\section{Discussion}

This study sought to preliminarily address the critical factors in an ICU setting (ie, need for sedative and analgesics, number of days on the ventilator, and ICU stay) by using nicotine replacement patches in the ICU setting with smokers. Nicotine replacement patches have been shown to be the most consistently effective NRT, when it is not coupled with adjuvant behavioral therapy, which is not feasible in the ICU. Importantly, NRT has been shown to be effective in hospitalized subjects in a meta-analysis of 17 double-blind, placebo-controlled studies. ${ }^{5}$ The nicotine patch delivers a constant rate of nicotine transdermally, which will not interfere with other ICU procedures (ie, sedation and/or ventilation procedures).

Though not statistically significant, our preliminary results demonstrate a trend toward decreased ICU stay with NRT. Although our data indicate that neither ventilator days nor days using analgesics, vasopressors, or sedation were significantly different between the NRT-treated and placebo groups, more subjects are needed to better evaluate whether the subjects' agitation levels contributed to their ICU stays.
In the general population, NRT is highly effective for smoking cessation, by limiting the side effects, including agitation, associated with smoking cessation. However, there are limited studies addressing its use in hospitalized subjects. ${ }^{1,6-8}$ Mayer and colleagues ${ }^{9}$ reported 5 cases of agitated delirium in smokers hospitalized for brain injury. The delirium developed 2-10 days after smoking cessation, and either completely resolved or substantially improved following NRT. In another report, Tran-Van et al ${ }^{10}$ described a subject with restlessness and difficulty being weaned from mechanical ventilation, which was attributed to nicotine withdrawal. The patient's condition improved following NRT.

Although most studies assess NRT as having a positive correlation with death, the literature reveals that the more pertinent outcome to measure is the need for additional sedatives and/or analgesics, and then correlating this need to measured agitation and the occurrence of adverse events. According to Woods et al, ${ }^{11}$ in a 2004 study, reducing ICU agitation not only benefits the patient by reducing the need for sedatives and decreasing the time on the ventilator, but also lowers treatment cost by minimizing the need for additional medications associated with minimizing patient agitation. Downstream consequences of these sedatives include lowered breathing frequency and increased chance of ICU-associated pneumonia. ${ }^{11-13}$

Additionally, studies have shown that younger patients are more likely to become agitated in the ICU. ${ }^{11}$ The median age in the present study was $57.4 \mathrm{y}$ in the NRT group and $52.3 \mathrm{y}$ in the no-NRT group. This age population is not noted for increased risk of agitation when in the ICU and on mechanical ventilation. Therefore, performing a focused study on NRT in a younger ICU population with the focus on preventing agitation and associated adverse events may reveal a greater benefit in the ICU.

Studies have demonstrated that the patients who sit up, ambulate, and interact with their support network have a lower mortality rate in the ICU. ${ }^{12,13}$ NRT is potentially another means to facilitate this active behavior in the ICU and to lessen the need for agitation-induced sedation. Our results indicate a trend in reducing ICU stay and decreasing the duration of mechanical ventilation.

Lucidarme and colleagues ${ }^{2}$ described the impact of nicotine abstinence on the clinical course of critically ill patients receiving mechanical ventilation for at least 48 hours. Their study included 144 subjects (44 smokers and 100 non-smokers) and showed active smoking to be an independent risk factor for agitation. However, a prospective randomized clinical trial of hospitalized smokers by Lewis and colleagues ${ }^{14}$ did not show any difference in mortality between the nicotine patch versus placebo. Because of the paucity of clinical trials demonstrating the benefit of NRT for smoking cessation in ICU patients, NRT is generally 
Table 3. Effect of Nicotine Replacement Therapy in ICU Subjects

\begin{tabular}{|c|c|c|c|c|c|c|c|c|c|c|}
\hline & \multicolumn{10}{|c|}{ Nicotine Replacement Therapy } \\
\hline & \multicolumn{3}{|c|}{ Yes } & \multicolumn{3}{|c|}{ No } & \multicolumn{3}{|c|}{ Difference } & \multirow{2}{*}{$P^{*}$} \\
\hline & $n$ & Mean \pm SD & Median & $n$ & Mean \pm SD & Median & $n$ & Mean \pm SD & Median & \\
\hline Age, y & 20 & $57.4 \pm 12.0$ & 58.0 & 20 & $52.3 \pm 13.3$ & 52.5 & 20 & $5.1 \pm 14.6$ & 2.0 & .12 \\
\hline APACHE II score & 20 & $14.3 \pm 9.7$ & 11.5 & 20 & $13.8 \pm 9.4$ & 12.0 & 20 & $0.5 \pm 1.1$ & 0 & .07 \\
\hline ICU stay, d & 20 & $4.5 \pm 3.8$ & 3.0 & 20 & $7.0 \pm 5.8$ & 5.5 & 20 & $-2.5 \pm 6.6$ & -1.0 & .08 \\
\hline Packs of cigarettes per day & 20 & $1.2 \pm 0.5$ & 1.0 & 20 & $1.0 \pm 0.4$ & 1.0 & 20 & $0.1 \pm 0.7$ & 0 & .50 \\
\hline Days on ventilator & 20 & $1.9 \pm 3.7$ & 0 & 20 & $3.5 \pm 5.3$ & 1.0 & 20 & $-1.6 \pm 6.3$ & 0 & .47 \\
\hline Days on analgesia & 20 & $1.1 \pm 2.6$ & 0 & 20 & $2.1 \pm 3.8$ & 0.5 & 20 & $-1.1 \pm 4.5$ & 0 & .26 \\
\hline Days on vasopressors & 20 & $0.7 \pm 1.6$ & 0 & 20 & $0.5 \pm 1.3$ & 0 & 20 & $0.2 \pm 1.9$ & 0 & .78 \\
\hline Days on sedation & 20 & $1.4 \pm 2.5$ & 0 & 20 & $2.7 \pm 4.6$ & 0.5 & 20 & $-1.4 \pm 5.3$ & 0 & .41 \\
\hline Years of smoking & 20 & $24.4 \pm 10.2$ & 25.0 & 20 & $23.3 \pm 10.7$ & 20.0 & 20 & $1.1 \pm 10.8$ & 0 & .79 \\
\hline
\end{tabular}

used exclusively by intensivists, to ease or prevent withdrawal symptoms.

Cartin-Ceba et al ${ }^{15}$ completed a prospective observational study of smokers admitted to the ICU, in which they sought to investigate the impact of NRT on critically ill subjects, including hospital mortality (primary outcome), delirium, and cumulative doses of sedation and analgesia (secondary outcomes). A total of 330 active smokers (older than 18 years of age) were evaluated, and 174 received NRT upon admission to the ICU. A total of 14 subjects from the group receiving NRT (7.8\%) died, as did 10 subjects from the non-NRT group (6.3\%): a difference that was not statistically significant $(P=.60)$. However, the NRT group did experience significantly more delirium than the non-NRT group (average ICU days $169 \mathrm{~d}$ [23\%] vs $75 \mathrm{~d}$ [13.1\%], $P \leq .001$ ). Their results showed that NRT was not associated with increased hospital mortality after adjustment for severity of disease, pack-years of smoking, and do-not-resuscitate status upon ICU admission (odds ratio $1.6,95 \% \mathrm{CI} 0.6-4.1, P=.35$ ). They noted that a randomized controlled trial was needed to really evaluate these risks and benefits.

A double-blind, placebo-controlled clinical trial by Warner et $\mathrm{al}^{16}$ reported that routine NRT was not beneficial in the management of perioperative nicotine withdrawal in smokers who underwent elective surgery. Lee and Afessa ${ }^{17}$ conducted a retrospective case-control study in which they identified 90 cases (smokers who received nicotine replacement in the first $24 \mathrm{~h}$ of their medical ICU admission) and 90 controls (smokers who did not receive nicotine replacement), matched for APACHE III score and age. Unadjusted mortality was $20 \%$ in those who received nicotine replacement, and $7 \%$ in those who did not. When adjusted for severity of illness and mechanical ventilation, those who received NRT still had significantly higher mor- tality (odds ratio 24.6, CI 3.6-167.6). They concluded that NRT was associated with increased hospital mortality in critically ill subjects. Although their findings are striking, it was a single-center, retrospective study.

Paciullo et al ${ }^{18}$ studied the impact of NRT on in-hospital mortality following coronary artery bypass graft surgery. Sixty-seven subjects who received NRT were matched with 67 current smokers in terms of baseline demographics and surgical procedures. Mortality was nonsignificantly higher in the NRT group versus the non-NRT smoker group (4.5\% vs $0 \%, P=.08)$. In a follow-up commentary to Lucidarme et al, ${ }^{2}$ Afessa and Keegan ${ }^{19}$ affirmed that, due to the paucity of data describing nicotine withdrawal in the critically ill, prospective studies are needed to address the issues of mortality, as well as prevention and treatment options.

Although this was a pilot study with a small sample size, the positive-trending results indicate further studies should be performed on a larger scale at this and other institutions, to broaden the population of this study. Despite having a broad protocol in which patients from the emergency department or the general medical floor could be included in the study, the majority of subjects in this study were admitted to the ICU from the emergency room; the one subject who was not admitted through the emergency room spent $\leq 1$ hour on the general medical floor. In future studies we aim to evaluate the patient population admitted from the general medical floor to the ICU to compare the results to our present study.

\section{Conclusions}

Data supporting the safety or harm of NRT in preventing nicotine withdrawal syndrome in patients admitted to the ICU are very limited. Although ICU stay and ventilator 


\section{Outcome of Nicotine Replacement Therapy in Patients Admitted to ICU}

days decreased in subjects receiving NRT, statistically the preliminary data demonstrated no significant benefit. Enrolling more subjects and increasing the study's power could help better define the role of NRT in smokers admitted to the ICU.

\section{ACKNOWLEDGMENTS}

We thank Po-Huang Chyou, Biomedical Informatics Research Center, Marshfield Clinic Research Foundation, for assistance with statistical analysis, and Marie Fleisner of the Office of Scientific Writing and Publication, Marshfield Clinic Research Foundation, for editing assistance.

\section{REFERENCES}

1. Honisett TD. Nicotine replacement therapy for smokers admitted to intensive care. Intensive Crit Care Nurs 2001;17(6):318-321.

2. Lucidarme O, Seguin A, Daubin C, Ramakers M, Terzi N, Beck P, et al. Nicotine withdrawal and agitation in ventilated critically ill patients. Crit Care 2010;14(2):R58.

3. Rigotti NA, Arnsten JH, McKool KM, Wood-Reid KM, Singer DE, Pasternak RC. The use of nicotine-replacement therapy by hospitalized smokers. Am J Prev Med 1999;17(4):255-259.

4. Rigotti NA, Munafo MR, Stead LF. Smoking cessation interventions for hospitalized smokers: a systematic review. Arch Intern Med 2008; 168(18):1950-1960.

5. Campbell IA, Prescott RJ, Tjeder-Burton SM. Transdermal nicotine plus support in patients attending hospital with smoking-related diseases: a placebo-controlled study. Respir Med 1996;90(1):47-51.

6. Fiore MC, Smith SS, Jorenby DE, Baker TB. Effectiveness of the nicotine patch for smoking cessation. A meta-analysis. JAMA 1994; 271(24):1940-1947.

7. Meine TJ, Patel MR, Washam JB, Pappas PA, Jollis JG. Safety and effectiveness of transdermal nicotine patch in smokers admitted with acute coronary syndromes. Am J Cardiol 2005;95(8):976-978.

8. Reid R, Pipe A, Higginson L, Johnson K, D'Angelo MS, Cooke D, Dafoe W. Stepped care approach to smoking cessation in patients hospitalized for coronary artery disease. J Cardiopulm Rehabil 2003; 23(3):176-182

9. Mayer SA, Chong JY, Ridgway E, Min KC, Commichau C, Bernardini GL. Delirium from nicotine withdrawal in neuro-ICU patients. Neurology 2001;57(3):551-553.

10. Tran-Van D, Hervé Y, Labadie P, Deroudilhe G, Avarguès P. [Restlessness in intensive care unit: think to the nicotinic withdrawal syndrome]. Ann Fr Anesth Réanim 2004;23(6):604-606. Article in French.

11. Woods JC, Mion LC, Connor JT, Virary F, Jahan, Huber C, et al. Severe agitation among ventilated medical intensive care unit patients: frequency, characteristics and outcomes. Intensive Care Med 2004;30(6):1066-1072.

12. Morris AC, Hay AW, Everingham K, McCulloch C, McNulty J, Brooks $\mathrm{O}$, et al. Reducing ventilator-associated pneumonia in intensive care: impact of implementing a care bundle. Crit Care Med 2011;39(10):2218-2224.

13. Fields LB. Oral care intervention to reduce incidence of ventilatorassociated pneumonia in the neurologic intensive care unit. J Neurosci Nurs 2008;40(5):291-298.

14. Lewis SF, Piasecki TM, Fiore MC, Anderson JE, Baker TB. Transdermal nicotine replacement for hospitalized patients: a randomized clinical trial. Prev Med 1998;27(2):296-303.

15. Cartin-Ceba R, Warner DO, Hays JT, Afessa B. Nicotine replacement therapy in critically ill patients: a prospective observational cohort study. Crit Care Med 2011;39(7):1635-1640.

16. Warner DO, Patten CA, Ames SC, Offord KP, Schroeder DR. Effect of nicotine replacement therapy on stress and smoking behavior in surgical patients. Anesthesiology 2005;102(6):1138-1146.

17. Lee AH, Afessa B. The association of nicotine replacement therapy with mortality in a medical intensive care unit. Crit Care Med 2007; 35(6):1517-1521.

18. Paciullo CA, Short MR, Steinke DT, Jennings HR. Impact of nicotine replacement therapy on postoperative mortality following coronary artery bypass graft surgery. Ann Pharmacother 2009;43(7): 1197-1202.

19. Afessa B, Keegan MT. Critical care support of patients with nicotine addiction. Crit Care 2010;14(3):155. 